\title{
Autoevaluación para la acreditación \\ en la Escuela Tezza: relato de experiencia
}

\author{
Felipe Armando Atúncar Quispe ${ }^{1}$, Felipa Gastulo Morante ${ }^{2}$
}

\begin{abstract}
Atúncar QFA, Gastulo MF. Autoevaluación para la acreditación en la Escuela Tezza: relato de experiencia. Cuid salud, jul-dic 2014; 1(2).
\end{abstract}

\section{RESUMEN}

En el Perú, las carreras y programas profesionales de universidades públicas y privadas están comprometidas en brindar un servicio educativo de calidad, que garantice en los futuros profesionales lograr que sean competentes y competitivos en sus campos laborales. Para la Escuela Tezza, la calidad de la formación que reciben los futuros profesionales de enfermería ha sido en todo momento prioridad y desde el 2003 emprende de manera voluntaria su proceso de autoevaluación con fines de mejora, para concretizar dicho proceso con la acreditación. Este articulo relata el proceso de preparación realizado por la Escuela durante los últimos 10 años camino hacia la acreditación.

Palabras clave: autoevaluación, acreditación, calidad, mejora.
Atúncar QFA, Gastulo MF. Self-assessment for accreditation in Tezza College: experience report. Cuid salud, jul-dic 2014; 1(2).

\begin{abstract}
In Peru, careers and professional programs of public and private universities are committed to provide quality educational services, to ensure in future professionals to make them competent and competitive in their work fields. For Tezza College, the quality of the training given to future nursing professionals has been a priority throughout time and since 2003 voluntarily undertakes the process of self -evaluation with improvement purposes, to concretize the process to accreditation. This article describes the preparation process executed by College during the past 10 years towards accreditation.
\end{abstract}

Keywords: Self-evaluation, accreditation, quality, improvement.

\footnotetext{
${ }^{1}$ Licenciado en Educación. Docente de la Escuela de Enfermería Padre Luis Tezza, afiliada a la Universidad Ricardo Palma, LimaPerú. ${ }^{2}$ Magíster Enfermera. Religiosa de la Congregación Hijas de San Camilo. Directora de la Escuela de Enfermería Padre Luis Tezza, afiliada a la Universidad Ricardo Palma, Lima- Perú
} 


\section{INTRODUCCIÓN}

La educación superior tiene retos trascendentales en el mundo globalizado y uno de ellos es la demanda de una educación superior de calidad, considerando que en los últimos años existe un aumento del número de universidades, tanto públicas como privadas, a nivel mundial y en el Perú, las cuáles compiten por proporcionar una educación de calidad. Este panorama muestra que la calidad en el sector educación está dando origen a diversos modelos, métodos, estándares, indicadores y criterios de evaluación que finalmente se concretiza con la acreditación de una carrera, programa o de una institución.

En los últimos años, se ha conceptualizado la calidad universitaria desde varios enfoques, adoptando diferentes modelos, los que finalmente coinciden en determinar el nivel de gestión, procesos de enseñanza-aprendizaje, docentes, investigación, proyección social, bienestar, infraestructura e, incluso, los recursos financieros que debe manejar una carrera o universidad; con el único propósito de formar profesionales competentes y capacitados para afrontar los problemas de la sociedad, comunidad, región, gobierno, empresas e instituciones que lo requieran.

Bajo esa perspectiva, se busca asegurar y promover la calidad de las carreras o programas de estudios en las universidades, aplicando mecanismos de autoevaluación, sea con fines de mejora o de acreditación; sobre esta última se requiere preferentemente de una evaluación externa, a fin de verificar si se cuenta o no con los requerimientos mínimos de calidad que debe cumplir una carrera o programa educativo.

Muchos países han mostrado interés por la calidad del servicio educativo que brindan las universidades a la sociedad, invirtiendo lo necesario $\mathrm{y}$, en algunos casos, lo indispensable para su mejora. Otros, han preferido adoptar una cultura de calidad educativa siguiendo algunos modelos, entre ellos destacamos a: Malcolm Baldrige ${ }^{1}$ de los Estados Unidos, el Total Quality Management (TQM) ${ }^{2}$ Sistema de calidad total, el modelo de la Organización Internacional de Normalización (Internacional Stándard

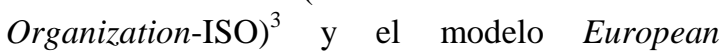
Foundation for Quality Management (EFQM). ${ }^{4}$

En general, los modelos de evaluación educativa de la calidad universitaria son producto de la evolución; inicialmente artesanales, luego industriales y por último, en serie, siendo en muchos casos coincidentes en el análisis de procesos y, al final, como retroalimentación de los mismos, los resultados. En los cinco continentes, la acreditación muestra algunas semejanzas y considerables diferencias; lo cierto es que hasta ahora los países interesados por la calidad de los servicios educativos han tomado dos opciones: algunos crearon un organismo estatal que pone las reglas, establece las dimensiones, crea estándares e indicadores y realiza la evaluación; otros, ponen las reglas y estándares, pero acreditan a empresas o equipos de personas que realizarán el estudio de evaluación. Pero, en ambos casos, consideran la acreditación de carácter temporal, que puede durar de 3 a 4 años con una inspección de calidad; que de ninguna manera tendrá carácter punitivo, pudiendo ser voluntaria u obligatoria.

Entre los países donde la acreditación es voluntaria estan: Colombia (CNA), ${ }^{5}$ Bolivia (CONAES), Costa Rica (SINAES), ${ }^{6}$ México $\left(\right.$ CIIES $^{7}$ y COPAES $),{ }^{8}$ Venezuela (CNU) y Paraguay (ANEAES). ${ }^{9} \quad$ Ya en Argentina (CONEAU), ${ }^{10}$ Uruguay, Ecuador (CEAACES), ${ }^{11}$ Chile $(\mathrm{CNA})^{12}$ y Brasil (CONAES), ${ }^{13}$ la acreditación es obligatoria, destacando que en Argentina este proceso se realiza desde 1955.

En los Estados Unidos de Norteamérica (USA) existen seis agencias acreditadoras institucionales y unas 50 de programas. En Europa se encuentra a la Agencia Nacional de Evaluación de la Calidad y Acreditación (ANECA), ${ }^{14}$ localizada en España y que acredita a toda la región de Europa, aunque también existen otras agencias acreditadoras de igual prestigio como es el caso de la European Association for Quality Assurance in Higher Education (ENQA), ${ }^{15}$ cuyos países miembros son: Gran Bretaña, Francia, Alemania, Bélgica, Holanda, Suecia, Dinamarca, Finlandia e Italia, entre los más destacados.

Estas instituciones acreditadoras emplean modelos de autoevaluación que se realizan mediante la apreciación de: 1) sólo procesos: aquí se evalúan a las carreras $\mathrm{y}$, a veces, a las instituciones (América Latina y Asia), 2) procesos y resultados: en ésta se evalúan a las carreras e instituciones (México y España) y, 3) resultados, en la que también puede considerarse los procesos; entre ellos se destaca aquellos que a) suele ser ejecutado por el Estado (se evalúa a las instituciones y las carreras que ofrece mediante un examen a los estudiantes. Ej: Brasil), b) puede ser realizado por el Estado y la actividad privada (se 
evalúan a las instituciones y las carreras que ofrece mediante un examen a los docentes. Ej: Países Noreuropeos) y, c) realizado solo por la actividad privada (se evalúa la eficiencia y calidad de la docencia e investigación mediante las investigaciones realizadas. Ej: Holanda. Se incluyen también aquí las publicaciones científicas. Ej: Finlandia, Suecia y Dinamarca).

Como se ha descrito, la acreditación ha sido un mecanismo practicado por muchos países con el propósito de medir la calidad de sus servicios y rendimiento, frente a estándares reconocidos por una institución o agencia acreditadora, siendo en todas sus formas un proceso que ha implicado la autoevaluación de la organización; en ese sentido, el objetivo de este relato es describir la experiencia del proceso de autoevaluación con fines de acreditación de la Escuela de Enfermería Padre Luis Tezza, afiliada a la Universidad Ricardo Palma, con la finalidad de socializar aspectos relevantes y sirvan de guía o referente para que otras instituciones formadoras emprendan un proceso similar, en aras de mostrar el cumplimiento de un grupo de estándares que garantizan la calidad del servicio brindado en un intervalo de tiempo.

\section{El proceso de acreditación en el Perú}

En el país, el Consorcio de Universidades Privadas del Perú, conformado por la Universidad Católica del Perú, Universidad Peruana Cayetano Heredia, Universidad del Pacífico y la Universidad de Lima, desde 1996 viene preparando modelos de evaluación con estándares de calidad y el 2005 publicaron el libro "Gestión de la Calidad para Instituciones de educación superior. Procesos de autoevaluación y acreditación", 16 que propone un modelo de autoevaluación en un marco de mejora continua que privilegia el mecanismo denominado autorregulación. Dicho modelo tomó como referencia modelos aplicados con éxito en otros países latinoamericanos; cuya metodología permite analizar la información recolectada cruzando y contrastando los resultados obtenidos, lo que facilita una visión global y transversal de toda la institución o carrera. Esta característica de análisis, denominada trazabilidad es un aporte significativo del modelo, de particular utilidad en la gestión de las instituciones de educación superior. Para que el proceso de autoevaluación logre sus objetivos, el modelo plantea incorporar la participación de todas las personas involucradas con la institución o carrera; así además de recoger información formal, toma en consideración la percepción y el grado de satisfacción de todos los actores vinculados con la unidad sujeta a evaluación. Finalmente, el modelo se adapta a las características de cada institución y se aplica tanto a procesos de autoevaluación institucional, como a programas o carreras.

Otra institución es la Asociación Peruana de Facultades de Medicina (ASPEFAM), creada en 1964 y que en 1999 hizo aprobar la Ley $27154^{17}$ que establece constituir la acreditación de las Facultades o Escuelas de Medicina Humana de las Universidades en el país, con la finalidad de garantizar la calidad e idoneidad de la formación académico-profesional de los médicos cirujanos y de la atención de la salud a la comunidad; asimismo, la Ley determina la creación de la Comisión para la Acreditación de Facultades o Escuelas de Medicina Humana (CAFME), cuyas funciones eran elaborar Normas para establecer los estándares mínimos de acreditación así como la administración del proceso, la supervisión y evaluación permanente. En enero de 2001 se aprueban los estándares mínimos, comenzando de inmediato el proceso de acreditación en los 28 centros académicos (12 privadas y 16 públicas) que existían en esa fecha para formar médicos. Ese mismo año se inician las visitas a las facultades para verificar el cumplimiento de los estándares; siendo que de las 19 facultades con una o más promociones de egresados, siete (cuatro públicas) consiguieron su acreditación el 2002 por cinco años; en tanto que las otras 12 (nueve públicas) lo hicieron el 2003. Las otras nueve no tenían estudiantes egresados cuando inició ese proceso, por lo tanto, la verificación del cumplimiento de los estándares se realizó según el grado de desarrollo de cada una. En dicha verificación se mostró que de las nueve, siete (cinco privadas y dos públicas) cumplían con los estándares; las dos restantes quedaron sujetas a nuevas verificaciones, inclusive, expuestas a que la CAFME solicitase a la Asamblea Nacional de Rectores la suspensión de nuevas inscripciones (estudiantes).

Entre los logros atribuidos al CAFME se destacan: 1) haber incorporado a las 28 facultades y escuelas de medicina, colectivos de médicos e instituciones de salud, al proceso de elaboración de los instrumentos de evaluación y acreditación; que, además, fueron prolijamente desarrollados; 2) estar impulsando la cultura de la calidad en los medios de la formación médica; 3) colocar en la agenda política y universitaria la necesidad de la acreditación y, 4) desarrollar criterios y procedimientos que sirven como antecedentes para la extensión de la acreditación a otras áreas 
profesionales. Sin embargo, es criticada porque no deja claro qué es acreditación y qué certificación; reduciéndose la acreditación solo al cumplimiento de requisitos que justifican la autorización o continuación de funcionamiento; por otro lado, sus estándares de calidad no son equivalentes con los internacionales, convirtiéndose en una instancia de legitimación de la insuficiencia y de la falta de idoneidad, dado que prácticamente acreditó a todas las facultades. Ínterin, cabe reconocer que se trata de la primera experiencia de acreditación en el Perú, la cual alimenta a los actuales procesos de acreditación.

En el 2002, la Asamblea Nacional de Rectores (ANR) -representante de las universidades de todo el país hasta febrero del 2015- crea la Comisión Nacional de Rectores para la Acreditación Universitaria (CNRAU), dicho organismo se encargaría de informar de todo lo relacionado con el proceso de mejoramiento continuo de la calidad en las Universidades, a través de la autoevaluación, evaluación y acreditación. Tal es así, que el 2005 propone un modelo de evaluación con fines de mejora, ${ }^{18}$ el cual contiene indicadores de evaluación y sus fuentes de verificación, además de modelos de encuestas para estudiantes, docentes, administrativos, egresados y grupos de interés.

La Accreditation Board for Engineering and Technology (ABET) fue creado en 1932 en USA para que las universidades contaran con un mecanismo que les permitiera verificar si estaban o no adecuándose a la acelerada evolución de las tecnologías. En ese sentido, el 2002 este organismo convoca a instituciones empresariales y académicas del país, para invitarles a integrar el Consejo Permanente de ABET-Perú, consejo que se constituiría en abril de ese mismo año, contando con las carreras de Ingeniería Electrónica de las universidades: Católica, Universidad Peruana de Ciencias Aplicadas y Universidad Nacional de Ingeniería.

En el 2006, se promulga la Ley N²8740 del Sistema Nacional de Evaluación y Acreditación y Certificación de la Calidad Educativa (SINEACE) $^{19}$ y, consecuentemente, el Consejo Nacional de Evaluación y Acreditación Universitaria (CONEAU-Perú), órgano operador encargado de definir los criterios, indicadores y estándares de medición para garantizar en las universidades públicas y privadas los niveles aceptables de calidad, así como alentar la aplicación de medidas para su mejoramiento.
El modelo de CONEAU-Perú cuenta con 3 dimensiones, 9 factores, 18 criterios de evaluación, 109 indicadores y 151 fuentes de verificación; todos en su conjunto permiten evaluar a las carreras profesionales en general, iniciando con las de educación y salud, luego se extenderían a las demás carreras, incluyendo a los programas y escuelas de postgrado. Aunque en agosto de 2014, el Estado Peruano promulga la nueva Ley Universitaria, quedando desarticulado los organismos operativos del SINEACE y entre ellos el CONEAU. Actualmente, sólo el SINEACE es responsable de garantizar a la sociedad que las instituciones educativas de educación superior ofrezcan servicios de calidad.

\section{Los inicios de la Escuela Tezza}

Desde su creación, la Escuela de Enfermería Padre Luis Tezza (EEPLT), adquirió el carácter universitario, afiliándose en sus inicios con la Universidad Femenina del Sagrado Corazón (UNIFE) y, desde 1988 a la fecha con la Universidad Ricardo Palma (URP).

La Escuela Tezza es una institución educativa católica que desarrolla un servicio de calidad de manera ininterrumpida, desde hace 31 años, a las jóvenes con vocación de servicio y deseos de realizarse profesional y personalmente. Fue creada por las Congregación Religiosa Hijas de San Camilo con el firme propósito de extender los 100 brazos de caridad hacia los enfermos, a través de las futuras enfermeras tezzianas.

En todo momento, la Escuela Tezza se ha caracterizado por formar profesionales de enfermería de alto nivel académico, científico, tecnológico y humanístico, con valores y principios éticos, bajo la mística camiliana que los hace únicos dentro de los profesionales del campo de la salud. La enfermera Tezziana en su formación universitaria responde a las exigencias del mercado laboral, pero también a la condición humana mediante su formación espiritual que le ofrece herramientas para cuidar al paciente bajo la espiritualidad de San Camilo de Lellis; ${ }^{20}$ además participa de actividades extracurriculares que refuerzan la mística camiliana para empoderarla del lema que regirá su quehacer profesional "cuidar al enfermo como lo hace una madre con su único hijo enfermo".

\section{La primera autoevaluación en la Escuela Tezza}

La Asociación Peruana de Facultades y Escuelas de Enfermería-ASPEFEEN emprendió 
desde el 2003 el Proyecto "Planificación e Implementación del Proceso de Autoevaluación y Acreditación de Programas de Pregrado en Facultades y Escuelas de Enfermería”, año en que se aprueban los Estándares Básicos de Calidad para los programas de Pregrado de Enfermería.

En ese contexto, la Escuela Tezza decide enrumbar, por primera vez, su proceso de autoevaluación fundamentado en los estándares básicos de ASPEFEEN, cuya estructura contenía nueve áreas, 32 variables y 68 indicadores, reconociéndose como áreas: la estructura académico administrativa, plan curricular, investigación, medios educativos, infraestructura física, personal docente, proceso de admisión, seguimiento de egresados y, proyección social y bienestar universitario. $^{21}$

Al emprender este proceso, la Escuela Tezza conforma su primera Comisión Central de Autoevaluación (2003), presidida por la religiosa Lic. Maria Luisa Chávez Salazar, siendo Coordinadora la Lic. Elga Sarmiento Bedoya y miembros activos: la religiosa Lic. Flor Barreda Correa y, los laicos, Mg. Angélica Aguirre de Chauca, Lic. Hilda Filinich de Zegarra, Lic. Milagros Salazar De la Torre y Lic. Maria Evelina Gastulo Morante. Paralelamente, fue aprobado su Reglamento de Acreditación, el cual constaba de 5 capítulos, 28 artículos y un anexo.

Se resalta que el 2004, ASPEFEEN puso énfasis al proceso de autoevaluación iniciando una campaña que denominó "Movilización Nacional por la Calidad de la Educación en Enfermería”, en la que participó activamente la Escuela Tezza. De ese modo, se realizaron diversas actividades, como las jornadas de capacitación dirigidos a los comités de autoevaluación a fin de motivar y sensibilizar a los docentes, administrativos y egresados de cada casa de estudio sobre los objetivos del proceso de autoevaluación y los avances que se venían logrando. En ese entonces, se elaboraron y aplicaron encuestas de satisfacción; además de producir documentos de gestión, actualización de reglamentos, implementación de programas y planes de mejora.

En noviembre de ese año, ASPEFEEN comunica a la Escuela que, habiendo realizado su proceso de autoevaluación, debe continuar con la siguiente fase y solicitar la visita de los Evaluadores Externos para corroborar lo informado y, a través de los resultados, proveer a la institución aportes para la mejora continua y el aseguramiento de la calidad de los Programas de Pregrado de Enfermería. Basado en esto, ASPEFEEN, a través de su comité de Evaluación y Acreditación designa una Comisión de Evaluación Externa, la cual fue responsable de ejecutar la visita durante tres días consecutivos desde el 30 de noviembre. Dicha visita contempló: reunión con las autoridades, revisión documentaria, entrevista con los docentes, administrativos y estudiantes, visita a las instalaciones, preparación del informe verbal preliminar y presentación del informe verbal preliminar a las autoridades; previamente, ASPEFEEN remitió a la Escuela Tezza dos documentos: un Procedimiento para la Evaluación Externa de Facultades/Escuelas de Enfermería y, un Código de Ética de los Evaluadores de Facultades/Escuelas de Enfermería.

Durante el proceso de autoevaluación, la Escuela Tezza revisó, actualizó y reformuló diversos documentos de gestión: Plan Estratégico Institucional, Manual de Organización y Funciones, Reglamento Institucional, entre los más importantes. Por otro lado, también fueron ordenados procesos claves utilizados para el desarrollo de la formación profesional referente a la enseñanza-aprendizaje, la investigación y la proyección social. Aspectos importantes que permitieron visualizar de modo integral la formación que se venía desarrollando y proyectar reformas substanciales con miras a una mejora continua de la calidad de la educación.

Finalmente, el 31 de enero del 2005 ASPEFEEN otorgó a la Escuela el documento de Aprobación de los Estándares Básicos de Calidad en la Autoevaluación de la Escuela Profesional de Enfermería, que describía el cumplimiento de los indicadores de las variables de los estándares básicos de calidad; además de contemplar un Programa de Mejora Continua de la Calidad basado en los resultados susceptibles de mejorar según los Pares Evaluadores. Los planes de mejora estaban dirigidos, específicamente, a mejorar el proceso de admisión, garantizar el seguimiento de los egresados, revisión permanente del currículo de estudio y a equipar y mejorar las condiciones de las aulas y laboratorios que se utilizan en la labor académica.

\section{La segunda autoevaluación en la Escuela Tezza}

La segunda experiencia se realizó con la Universidad Ricardo Palma-URP, liderado por el Rector Dr. Iván Rodríguez Chávez, que desde el 2003 había emprendido el camino hacia la excelencia para la mejora continua de la calidad y 
bajo la Dirección Ejecutiva del Dr. José Clemente Flores Barboza. Las autoridades de la URP tomaron la decisión de someter a todas las carreras de la universidad al proceso de autoevaluación, con la finalidad de generar un diagnóstico centrado en la gestión administrativa, la enseñanza, la infraestructura y el equipamiento tecnológico. Decisión que tenía como propósito destacar que la Universidad debía ser una opción preferente entre las otras universidades del país; de ese modo, se iniciaba una intensa actividad de equipo, cuyo fin era generar la normativa pertinente para construir el sistema organizativo, elaborar los estándares de calidad para la autoevaluación y disponer de los recursos necesarios.

Inicialmente, fue elaborada la base legal que daría sustento a la estructura del sistema de autoevaluación, considerando las funciones, facultades y responsabilidades de los órganos operativos, el modelo de calidad, los estándares y las etapas por las que atravesaría el proceso hasta la producción de planes de mejora y su cristalización en la práctica. ${ }^{22}$ Destacando que en todo momento el autoevaluación fue participativa.

Como la Escuela Tezza había emprendido su proceso de autoevaluación con ASPEFEEN, decide también hacerlo con la Universidad Ricardo Palma; de ese modo, en noviembre de 2003, la Universidad invita a la Escuela Tezza a integrarse al proceso con miras a elevar los estándares de excelencia en la formación de los profesionales; para lo cual solicitó nominar a los miembros del Comité de Autoevaluación de la EEPLT, además de establecer el presupuesto para garantizar el proceso de autoevaluación y participar en conferencias y talleres de autoevaluación (diciembre 2003, febrero y agosto 2004 y, febrero y marzo 2005), dónde se trataron aspectos conceptuales y metodológicos de la autoevaluación. En dichas actividades participaron ponentes del Perú y extranjero, abordando temas como sistemas latinoamericanos de autoevaluación y acreditación, experiencias del país en esas materias, etapas del proceso, rol de las autoridades, docentes y estudiantes, instrumentos para la colecta de datos y las escalas, los factores en juego para el cambio organizacional, entre otros. Es en ese contexto que la Escuela Tezza conducía simultáneamente dos procesos de autoevaluación, aunque con modelos distintos, pero con ciertas semejanzas en los a estándares.

Posteriormente, la URP crea la Comisión de Información y Normalización para el proceso de autoevaluación en la Universidad en todas las Carreras de Pregrado, Postgrado y Diplomado. Comisión que propone a cada carrera realizar un diagnóstico previo a la autoevaluación, a fin de que las autoridades de la unidad académica y el personal interno conozcan el estado de calidad de la carrera o sección; así siendo en octubre del 2004, la URP elabora una "Guía para el diagnóstico previo a la autoevaluación del pregrado".

En el 2005, la URP elabora una "Guía de Autoevaluación", ${ }^{23}$ la cual fue publicada por la Oficina de Desarrollo Académico, resultando un valioso documento para el Comité de Autoevaluación de la Escuela Tezza, pues le permitió emprender el proceso de autoevaluación y elaborar un plan de mejoramiento continuo para la carrera de enfermería; dado que el mismo contenía información específica sobre: un marco conceptual respecto a la autoevaluación, los principios que orientan la política de calidad, autoevaluación y acreditación en la URP; los objetivos de la guía; la organización del proceso; los estándares y ponderación para el pregrado, postgrado y diplomados; la metodología del trabajo; las etapas del desarrollo de la autoevaluación y, un glosario de términos.

Se resalta, que la guía tenía objetivos claros y precisos, orientados a la formulación del diagnóstico evaluativo de las actividades de docencia, investigación, extensión y gestión de la Universidad; suministrando información para elaborar un plan estratégico destinado a elevar la excelencia en el cumplimiento de sus fines, sentar las bases de una cultura organizacional dirigido a los futuros procesos de autoevaluación con fines de acreditación, contar con informes autoevaluativos y planes de mejora de las unidades académicas, integrar resultados al plan estratégico y al plan presupuestal institucional y, contar con una masa crítica de docentes y estudiantes que generen la autorregulación en forma espontánea.

Los estándares para la autoevaluación de las carreras de pregrado, que emplearía la Escuela Tezza, estaban estructurados en 9 áreas, 44 dimensiones, 94 variables y 345 indicadores, incluyendo las áreas de: planificación institucional, formación profesional y currículo, investigación, extensión y proyección universitaria, profesores, estudiantes, administración, infraestructura y equipamiento $\mathrm{y}$, egresados. 
El comité de autoevaluación de la Escuela Tezza participó activamente en las diversas reuniones organizadas por la Comisión de Información y Normalización de la Autoevaluación de la URP, a fin de comprender los aspectos metodológicos relacionados con la aplicación de los instrumentos y procesamiento de los resultados. En ese sentido, la URP elaboró 35 instrumentos de autoevaluación que permitieron emitir juicios de valor sobre los factores materia de análisis de calidad de los procesos formativos de las carreras en la URP, considerando lo más apropiado para cada caso de evaluación, tales como cuestionarios, lista de chequeo, informes especializados y escalas de actitudes tipo Likert; las que evaluaron las nueve áreas descritas anteriormente por tipo de sujetos: autoridades, docentes, especialistas en educación universitaria, trabajadores no docentes, egresados, estudiantes, empleadores externos, beneficiarios de proyección social universitaria $y$, usuarios de bienes $y$ servicios. Otro documento elaborado fue la "Guía para análisis y valoración de los estándares de autoevaluación", herramienta metodológica indispensable para procesar los resultados de los instrumentos de autoevaluación aplicados a las partes interesadas para la mejora de la calidad en cada carrera.

La participación de la Escuela Tezza en la autoevaluación con la URP fue permanente y constante, elaborando el Informe Autoevaluativo Preliminar, cuyo único objetivo fue medir la situación de la Escuela (en ese entonces) y establecer las acciones correctivas a través de los planes de mejora continua, específicamente en la difusión de la misión y visión de la Escuela, seguimiento del Plan de Desarrollo Institucional, evaluación del currículo de estudio, actualización del reglamento de evaluación del estudiante, instalación del comité de investigación y su reglamentación, elaboración del reglamento del programa de extensión y proyección universitaria, informes de los servicios prestados a la comunidad, revisión y actualización de las políticas y mecanismos de selección docente, elaboración, seguimiento y evaluación del programa de capacitación docentes anual, evaluación semestral de los docentes, mejoramiento del sistema de admisión, implementación del programa de tutoría para los estudiantes, adecuación del número de aulas según la población estudiantil, programación del mantenimiento de laboratorios, adquisición de material bibliográfico, incremento del número de computadoras para el laboratorio, revisión y actualización de documentos de gestión, elaboración del sistema de evaluación e incentivos para el personal y, reactivación de la comisión de seguimiento de los egresados; todos éstos fueron programados a mediano y largo plazo con el firme propósito de mejorar la calidad del servicio a los miembros de la comunidad educativa.

\section{Capacitación del Comité de Autoevaluación de la Escuela Tezza}

Una experiencia que fortaleció a los miembros del Comité Interno de la Escuela Tezza fue el continuo interés de sus autoridades para promover una cultura de mejoramiento continuo y de calidad; de ese modo el 2009 financia la participación de un grupo de docentes y personal académico-administrativo en el "Programa de Formación de Especialista en Autoevaluación Universitaria", promovido por la Dirección General de Investigación y Calidad Universitaria de la Asamblea Nacional de Rectores. El objetivo del Programa fue desarrollar competencias para la mejora sistémica de la calidad del sistema universitario, además de lograr que los participantes sean profesionales líderes, con vocación de servicio, con competencias certificadas para el diseño, promoción y ejecución de procesos de autoevaluación universitaria. Dicha capacitación se realizó entre abril y noviembre de 2009, el cual fue diseñado en 3 módulos: Bases de Calidad Universitaria, El Proceso de Autoevaluación y la Formulación de Planes de Mejora. La metodología considerada fue la semipresencial; en las sesiones presenciales hubo exposiciones y ronda de preguntas, en tanto que en las virtuales se incluyeron lecturas complementarias, ejercicios de refuerzo, foros $\mathrm{y}$ trabajos integradores, todo esto a través de la plataforma B-learning (moodle).

La participación en el Programa permitió contar con un equipo involucrado con el proceso de autoevaluación, disponiendo de herramientas metodológicas básicas para emprender el proceso. En ese entonces, el gobierno organizaba un sistema de evaluación, autoevaluación y acreditación a nivel nacional, a fin de establecer los modelos de calidad para las carreras universitarias en el Perú. Más adelante, se conformaría el Primer Comité Interno oficial frente al Consejo de Evaluación, Acreditación y Certificación de la Calidad de la Educación Superior Universitaria (CONEAU), constituido por el $70 \%$ de profesionales que participaron en el Programa de la ANR, hecho que garantizó la acreditación más adelante. 


\section{La Autoevaluación de la Escuela Tezza con fines de Acreditación}

La Escuela Tezza emprendió su primera experiencia de autoevaluación en el 2003, aunque desde sus inicios lo hizo con fines de mejora continua. Las experiencias de autoevaluación desarrolladas con ASPEFEEN y la URP permitieron ingresar a un proceso relativamente nuevo, en la que primero se debía "ordenar la casa" a través de la elaboración y actualización de documentos de gestión y normatividad, además de implementar programas, promover actividades de investigación y proyección social, documentar reuniones y actividades académicas, entre otras actividades relacionadas con planificación, diseño, ejecución y evaluación de las acciones realizadas.

En el 2009, la Escuela Tezza conformó su primer Comité Central de Autoevaluación para enrumbarse a la acreditación nacional; Comité que estuvo presidido por la religiosa Mg. Flor Barreda Correa, acompañada de un grupo de profesionales con carga docente-administrativa, más dos representantes de los estudiantes. Este equipo fue estructurado en subcomités tomando en cuenta los factores del modelo de calidad propuesto por el CONEAU; posteriormente se integrarían otros dos miembros al Comité, quienes el 30 de diciembre por Resolución Directoral Nº147-2009 constituyen el Comité Interno conforme lo establece la Guía para la Acreditación de Carreras Profesionales Universitarias del CONEAU. ${ }^{24}$ De esta manera, se oficializaba el proceso de autoevaluación con fines de acreditación de la carrera de enfermería en la Escuela, comprometiéndose a desarrollar las siguientes etapas: génesis del proceso, generación de información y elaboración del informe final.

Por su parte, la URP convocó el 10 de agosto de ese año, al personal docente de sus diversas facultades, entre ellas la Escuela Tezza, a participar del XXIV Ciclo de Capacitación Docente desarrollando temas relacionados con la Ley No 28740 del Sistema Nacional de Evaluación, Acreditación y Certificación de la Calidad Educativa-SINEACE y su Reglamento ${ }^{25}$ y, sobre el Modelo de Calidad para la Acreditación de Carreras Universitarias, cuyo propósito era socializar los últimos alcances del CONEAU sobre la acreditación.

En octubre del mismo año, esta institución convoca a reunión a las autoridades y miembros de cada comité de autoevaluación de las escuelas/facultades de enfermería de las universidades del país (públicas y privadas), a fin de socializar el nuevo Modelo de Calidad para la Acreditación de la Carrera Profesional de Enfermería, participando también de ésta representantes de ASPEFEEN. Dicho Modelo fue replicado por el Comité Interno a los subcomités de la Escuela Tezza, con el propósito de recabar sugerencias sobre los estándares y fuentes de verificación propuestas en el Modelo, las mismas que se alcanzaron al CONEAU. Finalmente, en noviembre el Comité Interno elabora un plan de trabajo para realizar un diagnóstico institucional sobre las actividades de gestión de la carrera, formación profesional y servicios de apoyo para la formación profesional. Sobre este plan fue elaborado el plan estratégico destinado a asegurar la calidad y la mejora continua de la EEPLT; proceso que se concretizó hasta diciembre del 2010, bajo el lema de "Uniendo esfuerzos hacia la Acreditación Universitaria".

Como parte del proceso, en enero de 2010 se realizó el primer taller de motivación y sensibilización para toda la comunidad educativa, tomando como referente el Nuevo Modelo de Calidad del CONEAU. Modelo oficializado en el diario "El Peruano" bajo la denominación de Modelo de Calidad para la Acreditación de la Carrera Profesional Universitaria de Enfermería, ${ }^{26}$ que consta de 98 estándares organizados en nueve factores y tres dimensiones. En consecuencia, los subcomités de trabajo se adecuaron al número de factores: 1) planificación, organización, dirección y control; 2) enseñanza-aprendizaje; 3) investigación; 4) extensión universitaria y proyección social; 5) docentes; 6) infraestructura y equipamiento; 7) bienestar; 8) recursos financieros y, 9) grupos de interés; cuyo propósito era evaluar el cumplimiento de los estándares en cada factor.

En mayo del 2010, la Escuela, a través de la URP, informa al CONEAU el inicio de su proceso de autoevaluación y de los miembros que componen su Comité Interno, aprobado por Acuerdo de Consejo Universitario $\mathrm{N}^{\circ} 1187-2010$. De tal modo, que en junio se presenta el Proyecto de Acreditación a la URP para su revisión, siendo aprobado en julio; posteriormente se solicita al CONEAU capacitar a los miembros del Comité Interno en los procedimientos propios de la acreditación, evento que se concretizó en un taller dirigido por el Dr. Milber Ureña el 12 de agosto, detallando aspectos metodológicos del proceso de autoevaluación definido por el CONEAU-Perú.

La segunda etapa, corresponde al proceso de autoevaluación propiamente dicho, el cual fue 
dirigido por el Comité Interno, que en todo momento se esforzó para que autoridades, docentes, administrativos, estudiantes, egresados y grupos de interés participen activamente, siendo asesorados por el Dr. José Flores Barboza, Director de la Oficina de Desarrollo Académico, Calidad y Acreditación de la URP. Durante esta etapa fue esencial la formación recibida en el Programa de Especialistas en Autoevaluación Universitaria de la ANR, al recolectar la información, elaborar instrumentos y procesar los datos, tomando como referente lo programado en el proyecto de acreditación presentado por la Escuela Tezza, de acuerdo a la Guía para la Acreditación de Carreras Profesionales Universitarias del CONEAU-Perú, dando cumplimiento así a las etapas correspondientes.

Una vez cumplida la etapa de génesis del proceso, la Escuela Tezza realiza la generación de información de los estándares durante el 2012; en este periodo el Comité Interno desarrolla la gran tarea de recopilar y preparar la información requerida para justificar el cumplimiento de los diversos estándares de calidad, elaborando formatos, registros, actas, informes, planes, programas, además de actualizar documentos de gestión, todos adecuados a las fuentes de verificación del Modelo de Calidad. ${ }^{26}$

Los documentos generados, en su mayoría eran fuentes de verificación que correspondían a estándares nominales, tales como documentos de gestión y de índole académico, normas, reglamentos, planes, informes, actas de reuniones, registros, entre los más importantes; otro grupo de documentos respondía a los estándares de tipo valorativo, los que tenían la apreciación de expertos y quienes debían elaborar un informe dando su juicio de valor sobre lo exigido por el estándar. Por otro lado, se elaboraron proyectos de implementación de diversos programas y sistemas, documentos que reunían una cierta cantidad de actividades relacionadas entre sí para cumplir con un objetivo; estos documentos respondían a los estándares sistémicos, que por tratarse de la primera acreditación era suficiente presentar una propuesta en cuya estructura debía verificarse actividades organizadas en etapas de: planificación, diseño, implementación, seguimiento y control, las que contaban con responsables, metas, plazos, indicadores y un presupuesto para garantizar su ejecución. Por último, estaban los documentos relacionados con los estándares de satisfacción, que fueron preparados para medir la percepción de los usuarios y, a partir de ellos, obtener resultados que permitan demostrar el cumplimiento del objeto de evaluación.

Una vez elaborada toda la información requerida para justificar el cumplimiento de cada uno de los estándares, el Comité de Calidad de la Escuela Tezza realizó una auditoría interna entre enero y marzo 2013, paso incluido en la guía para la acreditar una carrera, realizando seguimiento para que se levanten las observaciones generadas y, luego, elaborar el informe de cierre de la auditoría.

Sobre la encuesta de autoevaluación, ésta fue aplicada por primera vez en diciembre de 2011 a modo de diagnóstico y, entre abril y mayo del 2013, para la encuesta final (correspondiente al proceso de cambio). Las encuestas desarrollados por estudiantes, docentes y administrativos fue realizada a través del Sistema Virtual de Autoevaluación-Tezza (SVA-Tezza) utilizando la herramienta virtual Google Docs enlazado a la intranet de la Escuela; mientras que los egresados y grupos de interés lo hicieron directamente. Se elaboró además, un cuestionario para docentes siguiendo las normas de la Guía para la Acreditación de Carreras Profesionales Universitarias del CONEAU-Perú, el cual fue aplicado en junio de 2013 a través del SVA-Tezza. Este cuestionario fue diseñado y creado por el Comité del Sistema de Información y Comunicación de la Escuela Tezza, lo que permitió realizar enlaces (links) para que los docentes visualicen las fuentes de verificación correspondiente a los estándares del Modelo de Calidad y así emitir opinión con una afirmación de cumplimiento del estándar (Sí) o (No). Con estos resultados se generaron diversos documentos que fueron de utilidad para determinar el cumplimiento de los estándares. En esta etapa fue de relevancia aplicar las encuestas y cuestionarios bajo el SVATezza, tanto en su aplicación como en su procesamiento, disminuyendo los tiempos para la toma de decisiones e identificar la percepción de estudiantes, docentes, administrativos y egresadas respecto a las debilidades; facilitando la implementación de acciones correctivas o, cuando necesario; someterlos a un taller de discusión para buscar alternativas de solución.

Con los resultados de la encuesta y cuestionario, se realizó un taller de análisis y discusión de la información, como sugerido por el CONEAU, el 25 de junio 2013 analizándose la información del cuestionario aplicado sólo a 
docentes, las encuestas aplicadas a los grupos de interés, la evaluación del Comité Interno e informes de auditorías internas. Dando como resultado varios documentos que permitieron registrar los resultados, el grado de cumplimiento y las iniciativas de mejora.

Dicho taller fue conducido por el Comité Interno, que contó con más del $50 \%$ de docentes, administrativos, estudiantes y egresados que regularmente asistían a los comités de calidad. Allí fueron sometidos a discusión 12 estándares cuyos resultados mostraban cierta divergencia entre los estudiantes, docentes y administrativos, incluyendo: motivación para los estudiantes, docentes y administrativos; cursos electivos del plan del estudio; el $75 \%$ de titulados ha realizado tesis; carga lectiva de estudiantes; número de egresados por promoción de ingreso; hora lectiva y no lectiva del docente; formación profesional del docente; experiencia del docente; dominio de idioma del docente; proceso de selección, ratificación y promoción del docente; grado académico del docente; y uso de la biblioteca virtual.

Cada uno de los estándares observados fueron analizados y socializados con los asistentes al taller, aunque algunos de los participantes desconocían de la existencia de dicha información o cómo es que se aplicaban o desarrollaban en la vida académica, siendo necesario exponer el plan de mejora que, a corto o mediano plazo, ayudaría a alcanzar el cumplimiento del estándar. Finalmente, los asistentes dieron conformidad al cumplimiento de los estándares observados, procediéndose a levantar las observaciones.

De esta manera, el Comité Interno estaba listo para ingresar a la tercera etapa de la autoevaluación, la elaboración del Informe Final, proceso realizado entre julio-setiembre 2013, conteniendo la información del proceso de autoevaluación y sus resultados, así como los planes de mejora correspondientes. Los resultados se presentaron por nueve bloques, los que tenían relación con los factores del Modelo, cada bloque contenía la siguiente información: cumplimiento de los estándares, una breve justificación, las fuentes de verificación y una conclusión por factor; el informe final de autoevaluación fue entregado en físico y en versión digital, esta última se hizo para facilitar el acceso a las fuentes de verificación, con la finalidad de poder mostrar la documentación sustentatoria en formato PDF a través de enlaces (links); modalidad que facilitó a los evaluadores disponer de toda la documentación requerida para corroborar el cumplimiento de cada uno de los 98 estándares del Modelo para la Carrera de Enfermería. Todo el procesamiento de la data fue estructurada por el Comité del Sistema de Información y Comunicación (SIC) de la Escuela Tezza en coordinación con el Comité Interno, lográndose adjuntar un total de 532 archivos, entre resoluciones, planes, informes, encuestas, entrevistas gravadas, documentos de gestión, indicadores de gestión, entre otros documentos utilizados como fuente de verificación.

Culminado el informe final de autoevaluación, se procedió a realizar las coordinaciones con las entidades evaluadoras reconocidas por el CONEAU-Perú, a fin de concretizar la visita de los evaluadores externos, entre la terna propuesta, la institución eligió a la Empresa Evaluadora con Fines de Acreditación SAC (EEFIA). Proceso que se cumplió el 21, 22 y 23 de noviembre de 2013, siendo cinco los evaluadores externos, quienes eran especialistas en el área de enfermería. Previo a la visita de verificación, se estableció un programa de actividades, conformidad que fue dada por ambas partes, quedando establecido así: presentación protocolar, revisión documentaria, visitas a sedes de prácticas, visita a instalaciones de la Escuela y, las reuniones con representantes de los docentes, estudiantes, administrativos, egresados y grupos de interés; culminando el programa con la presentación de los resultados.

Al culminar el tercer día, los evaluadores dieron conformidad a 96 estándares, otros dos fueron observados respecto a su forma y omisión. La observación de omisión se refería a que uno de los documento no contenía en la versión digital el estándar correspondiente; mientras que en la de forma, el archivo digital no contenía la versión final del documento requerido; ambas observaciones se absolvieron ese mismo día con los documentos en físico, pero fue necesario registrarlas e informar oficialmente a la sede central de EEFIA dentro de los plazos establecidos en la guía de procedimientos del CONEAU-Perú.

Finalmente, el 26 de marzo de 2014, el SINEACE, a través de la Resolución de la Presidencia del Consejo Superior No 028-2014COSUSINEACE/P, aprobó la Acreditación de la Escuela de Enfermería Padre Luis Tezza afiliada a la Universidad Ricardo Palma, para el periodo de enero de 2014 a diciembre de 2016; de esta 
manera, la Escuela Tezza marcaba un hito importante y trascendental en la historia del Perú y en la historia de la Enfermería Peruana, siendo la Primera Carrera de Enfermería Acreditada en la ciudad de Lima y la segunda en el Perú, con estándares nacionales. Reconocimiento celebrado por la Escuela Tezza el 29 de mayo de 2014, en ceremonia especial y emotiva en la que participaron autoridades del SINEACE, Ex CONEAU, Universidad Ricardo Palma, Facultad de Medicina Humana de la URP, Congregación Hijas de San Camilo, Colegio de Enfermeros del Perú, Jefas de los diferentes departamentos de enfermería de los hospitales y sedes con las cuales se tiene convenio y, por supuesto, la comunidad tezziana: estudiantes, docentes, administrativos egresados y autoridades.

\section{Consideraciones finales}

Sin lugar a dudas, la EEPLT, a lo largo de sus 31 años de fundación, se ha ido perfilando como una institución educativa que forma profesionales

\section{Correspondencia:}

Felipe Armando Atúncar Quispe

Correo electrónico: felipe.atuncar@ correo.eeplt.edu.pe

\section{REFERENCIAS BIBLIOGRÁFICAS}

1. NIST: National Institute of Standards and Technology. Baldrige Performance Excellence Program [homepage]. Estados Unidos: NIST; 25 mar 2010 [actualizado 15 de sep 2014; citado 5 de mar 2015]. Disponible en: http://www.nist.gov/baldrige/

2. Calidad Total TQM. Sistema de Calidad Total [homepage]. San Francisco CA: Tangient LLC; 2005 [actualizado 2015; citado 6 de mar 2015]. Disponible en: https://calidadtotaltqm.wikispaces.com/Sistema $\underline{+\mathrm{de}+\text { Calidad+Total }}$

3. ISO: International Organization of Standarization [homepage]. Suiza: ISO.org; s.f. [actualizado s.f.; citado 6 de mar 2015]. Disponible en: http://www.iso.org/iso/home/standards.htm

4. EFQM. The EFQM Excellence Model [homepage]. Bruselas: EFQM.org; s.f. [actualizado s.f.; citado 6 de mar 2015]. Disponible en: http://www.efqm.org/the-efqm$\underline{\text { excellence-model }}$ de enfermería de calidad, y ello se refleja en la labor que vienen desarrollando sus egresadas en los diversos ámbitos laborales del campo de la salud, tanto a nivel nacional como internacional; especialmente porque la formación brindada a sus estudiantes es holística y humanizada, cultivando en todo momento esa mística que las hace únicas y diferentes a las otras enfermeras; dado que ese carisma las identifica como profesionales competentes para "Cuidar al enfermo como lo hace una madre con su único hijo enfermo".

Esta valiosa experiencia ha permitido a todos los miembros de la comunidad educativa enrumbar una cultura de calidad, la que repercute en una buena formación y en la calidad del servicio educativo que se brinda a los estudiantes, quienes al ser egresados se ven fortalecidos para afrontar los retos que la sociedad actual exige, lográndose de esta manera que la enfermera tezziana tenga un sello propio que la caracteriza, haciéndola única, más humana y con vocación de servicio.

5. CNA: Consejo Nacional de Acreditación [homepage]. Bogotá-Colombia: CNA, Ministerio de Educación Nacional; s.f. [actualizado s.f.; citado 6 de ene 2015]. Disponible en: http://www.cna.gov.co/1741/channel.html

6. SINAES: Sistema Nacional de Acreditación de la Educación Superior [homepage]. Costa Rica: SINAES; 2014 [actualizado 2014; citado 6 de ene 2015]. Disponible en: http://www.sinaes.ac.cr/index.php?option=com content $\&$ view $=$ article $\&$ id $=20 \&$ Itemid $=101$

7. CIIES: Comités Interinstitucionales para la Evaluación de la Educación Superior [homepage]. México-DF: CIIES; s.f. [actualización s.f.; citado 6 de ene 2015]. Disponible en: http://www.ciees.edu.mx/

8. COPAES: Consejo para la Acreditación de la Educación Superior [homepage]. MéxicoDF; s.f. [actualizado feb 2015; citado 6 de mar 2015]. Disponible en: http://www.copaes.org/ 
9. ANEAES: Agencia Nacional de Evaluación y Acreditación de la Educación Superior [homepage]. Asunción: ANEAES, Gobierno Nacional; s.f. [actualizado s.f.; citado 6 de ene 2015]. Disponible en: http://www.aneaes.gov.py/aneaes/

10. CONEAU: Consejo Nacional de Evaluación y Acreditación Universitaria [homepage]. Buenos Aires-Argentina: CONEAU; s.f. [actualizado s.f.; citado 6 de ene 2015]. Disponible en: http://www.coneau.gov.ar/CONEAU/

11. CEAACES: Consejo de Evaluación, Acreditación y Aseguramiento de la Calidad de la Educación Superior [homepage]. QuitoEcuador: CEAACES; s.f. [actualizado 2014; citado 12 de ene 2015]. Disponible en: http://www.ceaaces.gob.ec/sitio/

12. CNA-Chile: Comisión Nacional de Acreditación [homepage]. Santiago: CNA; s.f. [actualizado 2014; citado 6 de ene 2015]. Disponible en: https://www.cnachile.cl/Paginas/Inicio.aspx

13. CONAES: Comissão Nacional de Avaliação da Educação Superior [homepage]. Brasília: Ministerio da Educação; s.f. [actualizado 2013; citado 01 de dic 2014]. Disponible en: http://portal.mec.gov.br/index.php?option=com content $\&$ view $=$ article $\&$ id $=16735 \&$ Itemid $=88$ $\underline{2}$

14. ANECA: Agencia Nacional de la Evaluación de la Calidad y Acreditación [homepage]. Madrid: ANECA; s.f. [actualización s.f.; citado 11 de mar 2015]. Disponible en: http://www.aneca.es/

15. ENQA: European Association for Quality Assurance in Higher Education [homepage]. Brussels: ENQA; s.f. [actualizado ago de 2014; citado 13 de mar 2015]. Disponible en: http://www.enqa.eu/

16. Consorcio de Universidades. Gestión de la Calidad para Instituciones de educación superior. Procesos de autoevaluación y acreditación. Lima: Consorcio de Universidades; 2005.

17. Ley que institucionaliza la acreditación de facultades o escuelas de medicina. Ley 27154.
Diario oficial El Peruano, Año XVII-N6957, (11 Julio 1999).

18. Asamblea Nacional de Rectores. Modelo de Autoevaluación con fines de mejora de las carreras universitarias. Lima: Dirección General de Investigación y Acreditación Universitaria, ANR; 2005.

19. Ley del Sistema Nacional de Evaluación, Acreditación y Certificación de la Calidad Educativa. Ley 28740. Diario Oficial el Peruano, año XXIII-Nº475, (23 May 2006).

20. Villa J. Una vida al servicio de los enfermos: San Camilo. $2^{\mathrm{a}}$ ed. Santafé de Bogotá: Kimpres Ltda; 1996. 120 p.

21. Asociación Peruana de Facultades y Escuelas de Enfermería (ASPEFEEN). Planificación e Implementación del Proceso de Autoevaluación y Acreditación de Programas de Pregrado en Facultades/Escuelas de Enfermería. Estándares Básicos de Calidad para los Programas de Pregrado de Enfermería. Lima; Enero 2003.

22. Flores JC. La experiencia de la autoevaluación: Base para la mejora de la acreditación nacional. Lima: Universidad Ricardo Palma; 2014.

23. Universidad Ricardo Palma (URP). Guía de Autoevaluación. Lima:URP; 2005.

24. CONEAU. Guía para la Acreditación de Carreras Profesionales Universitarias del CONEAU. Lima: El Peruano; 2009.

25. SINEACE. Reglamento de la Ley No 28740 , Ley del Sistema Nacional de Evaluación, Acreditación y Certificación de la Calidad Educativa. Lima: El Peruano; 2007.

26. CONEAU. Modelo de Calidad para la Acreditación de la Carrera Profesional de Enfermería. Lima: El Peruano; 2010. 